\title{
DEMULSIFIER SELECTION FOR WATER SEPARATION FROM HEAVY CRUDE OIL EMULSIONS OF IRANIAN OIL FIELD
}

\author{
Fatemeh Yazdanmehr ${ }^{1}$, Iulian Nistor ${ }^{2}$ \\ ${ }^{1}$ National Iranian Oil Company, PEDEC, Iran, Petroleum - Gas University of Ploiesti (PhD \\ Student), Romania \\ ${ }^{2}$ Universitatea Petrol-Gaze din Ploieşti, Romania \\ e-mail: fyazdanmehr@pedec.net
}

DOI: 10.51865/JPGT.2021.01.06

\begin{abstract}
In this study, various demulsifiers have been chosen for emulsion separation from heavy Iranian oil. The 16 types of water and oil based demulsifiers were tested using the selection procedure. Further, the current study assessed the effect of parameters like concentration, water cut and residence time on demulsification. Water emulsion separation has been found to be improved with selected oil-based demulsifiers. The results show that the oil based demulsifiers with a $\mathrm{pH}$ between 7-8 and dosage more than $100 \mathrm{ppm}$ have more dehydration efficiency.

The efficiency of water-based demulsifiers is lower than that of oil-based demulsifiers. Using the Qualitec-4 software, the possibility of increasing the efficiency of the former was investigated. The results show that the selection of water-based demulsifiers, with $\mathrm{pH}=7.76$, dose $=100$, time $=600$, had the best performance.
\end{abstract}

Keywords: emulsion, demulsification, water oil separation, demulsifiers, oil base, water base, Qualitec-4

\section{INTRODUCTION}

Oil-water emulsions were formed in the process of crude oil production, which is almost associated with water. During operation, a certain percentage of water was often distributed through the crude oil produced in the form of small drops. Oil operators must avoid the formation or breakage of these emulsions in order to reduce the impact on the operating process and in the alternative on environmental issues.

Demulsification is vital in the processing of crude oil, used in the process of removing the content of water and salts from them. The water is distributed in the form of fine drops of oil, and most salts are contained in the water. Because water contains salts, dehydration and desalination technology were applied simultaneously. Let's ssuppose contaminants, such as salt and water that are present in crude oil, have not been removed. In this case, there is a potential for severe corrosion and clogging problems in 
downstream installations etc. [1]. Crude oil produced in most fields of the world contains a significant amount of water and salts, and crude oil produced in the fields of Iran is no exception.

Regarding those mentioned above, the separation of the water emulsion is mandatory, but strong oil desalination of crude oil is required in order to balance the export oil cost and standard oil specification. Also, increasing water and brine by about $1 \mathrm{ppm}$ (part per million), reduces the oil price by around $0.85-1.3 \$$ for each barrel. To date, several techniques have been used such as the use of emulsifiers, gravitational separation or centrifugal decantation, $\mathrm{pH}$ modification, filtration, oil heating, separating membranes and electrostatic desalination. The percentage of associated water after processing must be less than $0,1-0,5$ per cent in most crude oil-water mixture treatment processes according to crude oil marketing specifications.

Because of the immiscibility between two liquids, demulsification oil is challenging. The mixing effect between the surfactants produced and existing on crude oil also causes the formation of emulsions. [2]. Naturally, emulsifying formations were located in the polar molecules of crude oil with a higher molar weight [3]. This includes resins, asphaltenes and organic acids (soluble in crude oil, such as naphthenic, carboxylic oil, etc.) and bases which are the main parts of the interfacial layer that determine the stabilized emulsion.

Also, some other factors that impact interfacial film stability are the $\mathrm{pH}$ of the water and the additive content [4]; however, for various oil sources, these results indicate different effect [5]. For example, Daaou et al. (2011) investigated the pH effect on Algerian oil emulsions and proposed a neutral medium with greater efficiency than the acidic or basic environment to stabilize the emulsions [6]. Fourtuny et al. (2007) studied the salinity impacts, water content, temperature and $\mathrm{pH}$ on the oil emulsions stability based on microwave processing. They observed which the demulsification efficiency is high in emulsions with high water content, except when high $\mathrm{pH}$ and salt content are involved simultaneously. It is shown that in emulsions with high water content, the demulsification rate is high, unless the $\mathrm{pH}$ and salt content were simultaneously high. [7]. In addition, by evaluation the droplet-size distribution described by an optical microscopy method, Moradi et al. (2011) studied the salinity impact on crude oil-water emulsions and noted that emulsions are more stable at lower aqueous phase ionic strength [8].

In the current research, the focus is on the investigation of demulsifiers $\mathrm{pH}$ effect on one of Iranian heavy crude oil emulsions stability. In order to improve the efficiency of water separation, a wide range of influence of chemical emulsifiers (water-based / oilbased) on the emulsion is available.

Qualitec-4, experimental design software, has been applied to obtain an optimized demulsifier for water separation. The effects of demulsifier type, residence time and injection dosage in the range of various water cuts have studied.

\section{1- PROCESS, SUBSTANCES AND MATERIAL DESCRIPTION}

\section{1-1- Crude oil characterization}

The crude oil desalting specification is shown in Table 1. In order to find the best desalination efficiency, it is important to optimize the design parameters such as 
temperature, electric field, demulsifiers, etc.

In this research, efforts have been taken to minimize the demulsifier volume and residence time in one of the existing Iranian heavy oil production units' desalination. The operation conditions are given in Table 2.

Table 1. Crude oil characterization

\begin{tabular}{|l|c|}
\hline \multicolumn{1}{|c|}{ Parameter } & Value \\
\hline Original water cut, \% & $2.37-2.45$ \\
\hline Pour point, ${ }^{\circ} \mathrm{C}$ & -24 \\
\hline Relative density $\left(25^{\circ} \mathrm{C}\right)$ & 0.9430 \\
\hline Viscosity $\left(20{ }^{\circ} \mathrm{C}\right), \mathrm{mPa} \cdot \mathrm{s}$ & 604.67 \\
\hline Sulphur content, \% & 4.35 \\
\hline Acid number, $\mathrm{mg} \mathrm{KOH} / \mathrm{g}$ & 0.37 \\
\hline Wax content, \% & 1.84 \\
\hline Asphaltenes content, \% & 12.79 \\
\hline Resin content, \% & 36.55 \\
\hline Ash content, $\%$ & 0.416 \\
\hline Initial boiling point, ${ }^{\circ} \mathrm{C}$ & 100 \\
\hline Wax precipitation point, ${ }^{\circ} \mathrm{C}$ & 19.1 \\
\hline
\end{tabular}

Table 2. Range of Operating Parameter

\begin{tabular}{|l|c|c|}
\hline \multicolumn{1}{|c|}{ Parameter } & Value & Unit \\
\hline Water cut & $2 \%-40 \%$ & Volume \\
\hline Chemical content & $20-200$ & ppm \\
\hline Residence time & $15-1200$ & minute \\
\hline Temperature & 95 & ${ }^{\circ} \mathrm{C}$ \\
\hline
\end{tabular}

Table 3. Demulsifier specifications

\begin{tabular}{|l|c|c|}
\hline Demulsifier specification & Value & Unit \\
\hline $\mathrm{pH}$ & $3-10$ & - \\
\hline Viscosity & $13-270$ & $\mathrm{mPa} . \mathrm{s}$ \\
\hline Flash point & $22-80$ & $\mathrm{~g} / \mathrm{cm}^{3}$ \\
\hline
\end{tabular}

The American standards (ASTM) have used for measuring the oil density (ASTM D 1298, 2001), the water density (ASTM D 362, 2001), the based and sediments and water (BS\&W) (ASTM D4007-2016) and the salt content (ASTM D 326, 1997).

The original water cut of a crude oil sample is given in Table 1. The crude oil characteristics and its properties have been tested in RIPI (Research Institute of Petroleum Industry) laboratory in IRAN. Also the study has been done based on different water cuts.

The emulsion's stability is depended on the properties of the oil and water and the interfacial film behavior. The stability of the emulsion is also influenced by external factors such as temperature and electrical field. In this study, the emulsion stability was investigated studied by gathering experimental data showing the water separated amount from the emulsion and the water and oil interfacial tension by adjusting the demulsifier concentration, type and residence time. Some of the characteristics of the demulsifiers used in the research are listed in table 3. 


\section{1-2-Materials}

Table 4 have demonstrated the chemicals used in this study. Most of the chemicals have supplied from SEMNAN, CEDEC, KING, and FALIZAN companies.

According to ASTM D 4006-81 "Standard Test Method for Water in Crude Oil by Distillation", the water cut of emulsions was tested by distillation.

Table 4 Type of chemical demulsifiers

\begin{tabular}{|c|c|c|c|}
\hline No. & Name & Base & Supplier \\
\hline 1 & Dw-1 & Water & SEMNAN \\
\hline 2 & Dw-2 & Water & SEMNAN \\
\hline 3 & Dw-3 & Water & KING \\
\hline 4 & Dw-4 & Water & KING \\
\hline 5 & Dw-5 & Water & CEDEC \\
\hline 6 & Dw-6 & Water & FALIZAN \\
\hline 7 & Dw-7 & Water & FALIZAN \\
\hline 8 & Dw-8 & Water & FALIZAN \\
\hline 9 & Do-1 & Oil & SEMNAN \\
\hline 10 & Do-2 & Oil & FALIZAN \\
\hline 11 & Do-3 & Oil & KING \\
\hline 12 & Do-4 & Oil & KING \\
\hline 13 & Do-5 & Oil & KING \\
\hline 14 & Do-6 & Oil & CEDEC \\
\hline 15 & Do-7 & Oil & FALIZAN \\
\hline 16 & Do-8 & Oil & FALIZAN \\
\hline
\end{tabular}

\section{1-3-Emulsion Preparation}

The reference method (SPE 65041) "Development of New green Demulsifiers" was used in oil production a centrifuge tube functioned as a dehydration vessel according to the test conditions. Based on reference method, the Water/Oil emulsion was made up of sampled crude oil from well. Formation water was used and then the mixture of water and crude oil was preheated. Withdrew simulated formation water and crude oil at different proportion according to water cut in emulsion and also 1000 RPM was applied as a Stirring rate.

\section{1-4-Screening of the Demulsifiers}

The screening method has carried out at constant $\mathrm{pH}$ and temperature. Different demulsifiers (oil/water base) concentration and residence time were applied for other water cut crude oil. The experimental data has analyzed, and the most effective demulsifier was determined based on water separation efficiency in the existing emulsion system in RIPI laboratory. The kinds of demulsifiers, including water base and oil base, have tested for a heavy crude sample from an Iranian oil field. The specification and type of tested oil/water base demulsifiers have shown in Tables 5 and 6. 
Table 5 Water based demulsifiers specification

\begin{tabular}{|c|c|c|c|c|c|}
\hline Name & $\mathrm{pH}$ & $\begin{array}{c}\text { Viscosity } \\
(\mathrm{mPa} . \mathrm{s})\end{array}$ & $\begin{array}{c}\text { Density } \\
\left(\mathrm{g} / \mathrm{cm}^{3}\right)\end{array}$ & $\begin{array}{c}\text { Flash point } \\
\left({ }^{\circ} \mathrm{C}\right)\end{array}$ & $\begin{array}{c}\text { Solidification } \\
\text { point }\left({ }^{\circ} \mathrm{C}\right)\end{array}$ \\
\hline $\mathrm{Dw}-1$ & 7.76 & 65.90 & 0.985 & 28.1 & $<-15$ \\
\hline $\mathrm{Dw}-2$ & 3.22 & 2.40 & 1.011 & 58.0 & -1 \\
\hline $\mathrm{Dw}-3$ & 6.28 & 270.30 & 1.018 & 80.1 & $<-5$ \\
\hline $\mathrm{Dw}-4$ & 6.36 & 184.60 & 1.018 & 36.1 & $<-5$ \\
\hline $\mathrm{Dw}-5$ & 8.10 & 61.20 & 0.984 & 22.2 & $<-5$ \\
\hline $\mathrm{Dw}-6$ & 10.38 & 147.60 & 0.999 & 55.1 & $<-5$ \\
\hline $\mathrm{Dw}-7$ & 10.91 & 14.80 & 0.995 & 57.6 & $<-5$ \\
\hline $\mathrm{Dw}-8$ & 5.44 & 34.10 & 1.042 & $>85$ & $<-5$ \\
\hline
\end{tabular}

Table 6 Oil based demulsifiers specification

\begin{tabular}{|c|c|c|c|c|c|}
\hline Name & $\mathrm{pH}$ & $\begin{array}{c}\text { Viscosity } \\
(\mathrm{mPa} . \mathrm{s})\end{array}$ & $\begin{array}{c}\text { Density } \\
\left(\mathrm{g} / \mathrm{cm}^{3}\right)\end{array}$ & $\begin{array}{c}\text { Flash point } \\
\left({ }^{\circ} \mathrm{C}\right)\end{array}$ & $\begin{array}{c}\text { Solidifying } \\
\text { point }\left({ }^{\circ} \mathrm{C}\right)\end{array}$ \\
\hline Do-1 & 4.05 & 66.61 & 0.914 & 53.1 & $<-5$ \\
\hline Do-2 & 7.59 & 42.95 & 0.948 & 65.2 & $<-5$ \\
\hline Do-3 & 7.17 & 102.21 & 1.002 & 22.2 & $<-8$ \\
\hline Do-4 & 7.35 & 112.43 & 0.988 & $>85$ & $<-5$ \\
\hline Do-5 & 8.90 & 247.83 & 1.012 & 57.1 & -4.5 \\
\hline Do-6 & 6.70 & 25.46 & 0.938 & 32.1 & $<-5$ \\
\hline Do-7 & 7.88 & 13.20 & 0.997 & $>80$ & $<-5$ \\
\hline Do-8 & 7.10 & 225.69 & 0.985 & 71.2 & $<-8$ \\
\hline
\end{tabular}

2- RESULTS AND DISCUSSION

\section{2-1- Oil / Water demulsifiers, base demulsifiers effect}

In this part of the study, the optimal conditions for removing water from heavy crude oil were found following laboratory tests. The sample was heated with an adequate heating bath to meet the temperature. At a specific temperature, the demulsifiers have injected into the emulsion.

\section{- Oil base demulsifiers}

The eight types of oil base demulsifiers (mentioned in table 4) have been tested. Each type of demulsifier is evaluated with the dosage of 50ppm, 100ppm, 150ppm, and $200 \mathrm{ppm}$. The experimental data of dehydration efficiency in different water cuts $(2 \%$, $7.8 \%, 20 \%$, and $40 \%$ ) with above-mentioned dosage for oil base demulsifiers are collected. The dehydration efficiency changes versus different demulsifiers dosage in constant water cut (7.8\%) at different residence time are reflected in the figures 1-4.

The result shows that when the dose was over 100 ppm, the use of Do-1 demulsifier was most effective.

When dosage was below 120ppm, the use of demulsifiers Do-5 and Do- 8 was the highest demulsifier efficiency. Demulsifier efficiency were over $96 \%$ when dosage at $200 \mathrm{ppm}$ of Do-1 and dosage at 100ppm of Do-5 \& Do-8. So the demulsifiers Do-5, Do8 and Do- 1 have been chosen as three of best oil base demulsifiers. Optimal dosages are $100 \mathrm{ppm}, 100 \mathrm{ppm}, 200 \mathrm{ppm}$ respectively. This study shows the oil base demulsifiers with $\mathrm{pH}$ between 7-8 and dosage more than $100 \mathrm{ppm}$, have similar behavior. In addition, oil base demulsifiers with high viscosity and high $\mathrm{pH}$ have more dehydration efficiency. 


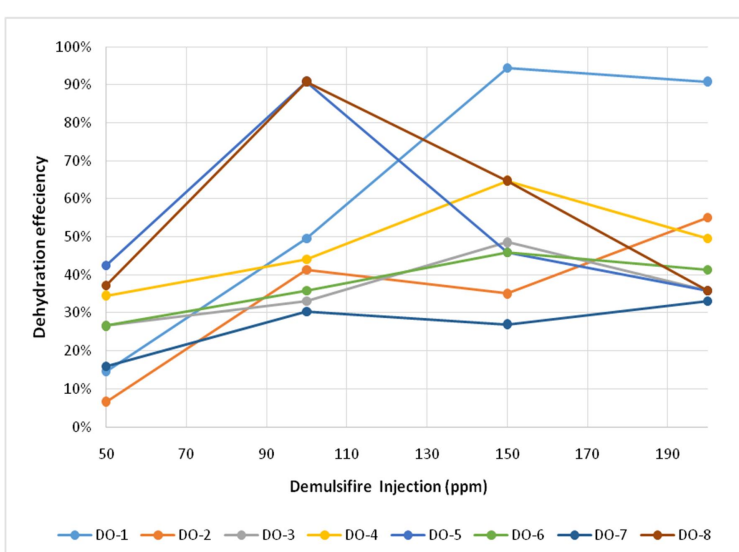

Figure 1. Dehydration efficiency of oil base demulsifiers for a residence time of 120 minutes

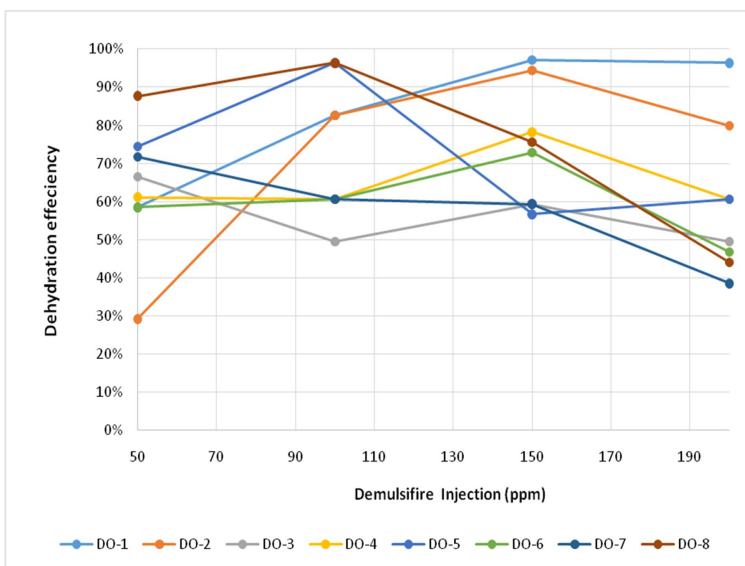

Figure 3. Dehydration efficiency of oil base demulsifiers for a residence time of 360 minutes

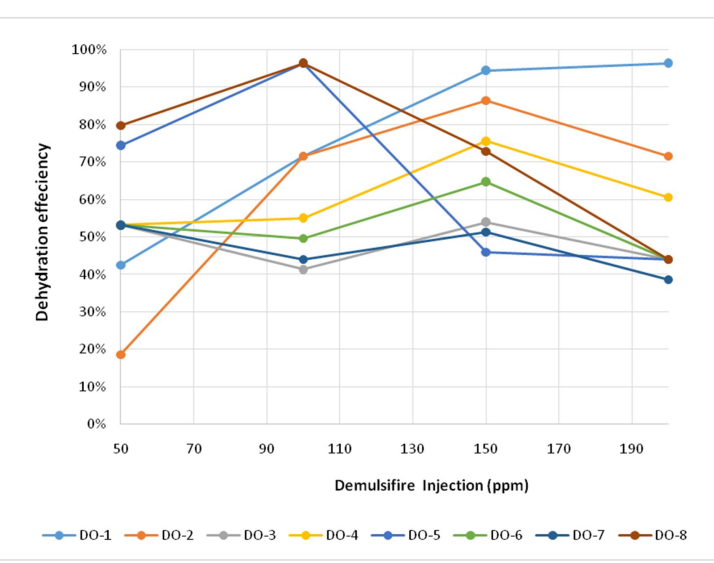

Figure 2. Dehydration efficiency of oil base demulsifiers for a residence time of 240 minutes

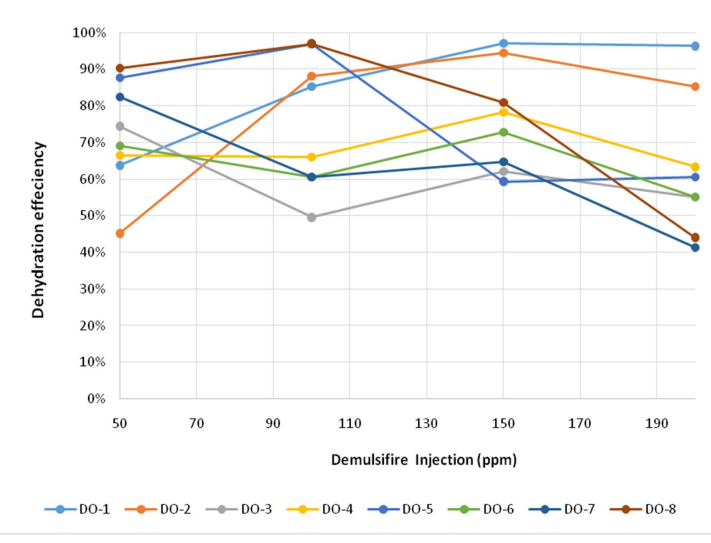

Figure 4. Dehydration efficiency of oil base demulsifiers for a residence time of 480 minutes

\section{- Water base demulsifiers}

Following the research, 8 types of water-based demulsifiers were tested. Each kind of demulsifier is evaluated with the dosage of $50 \mathrm{ppm}, 100 \mathrm{ppm}, 150 \mathrm{ppm}$, and $200 \mathrm{ppm}$. The experimental data of dehydration efficiency in different water cuts $(2 \%, 7.8 \%, 20 \%$, and $40 \%$ ) with above-mentioned dosage for water base demulsifiers are collected. The results of water base demulsifiers efficiency versus different dosage in constant water cut $(7.8 \%)$ at different residence time are reflected in the figures $5-8$. 


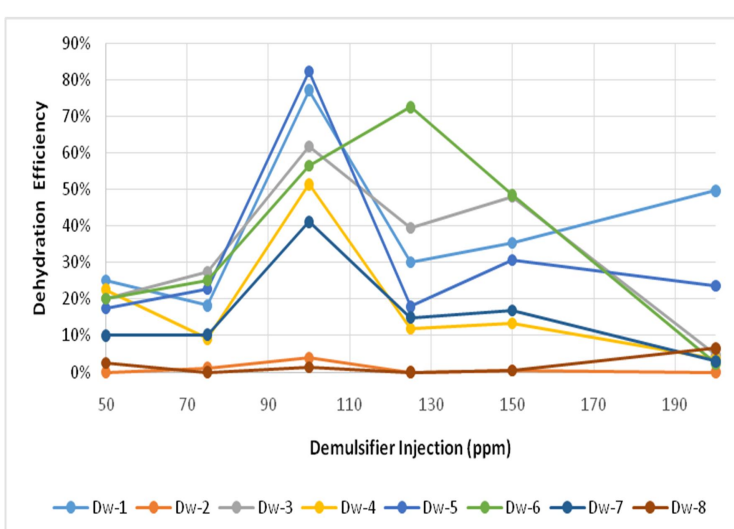

Figure 5. Dehydration efficiency of water base demulsifiers for a residence time of 120 minutes

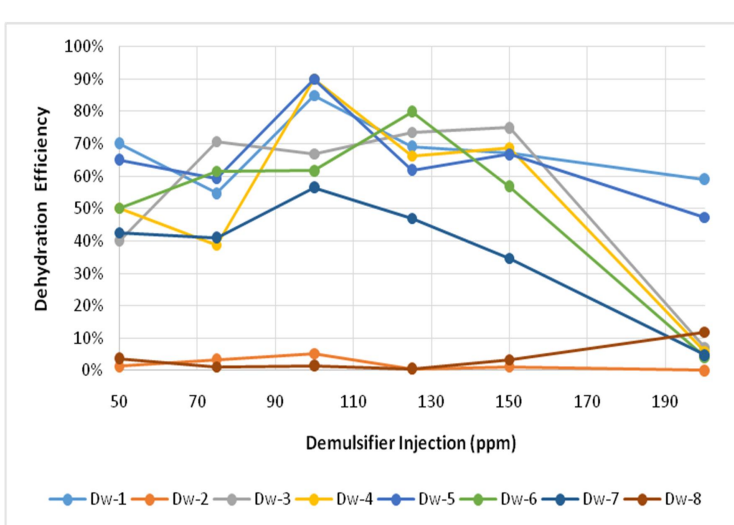

Figure 7. Dehydration efficiency of water base demulsifiers for a residence time of 360 minutes

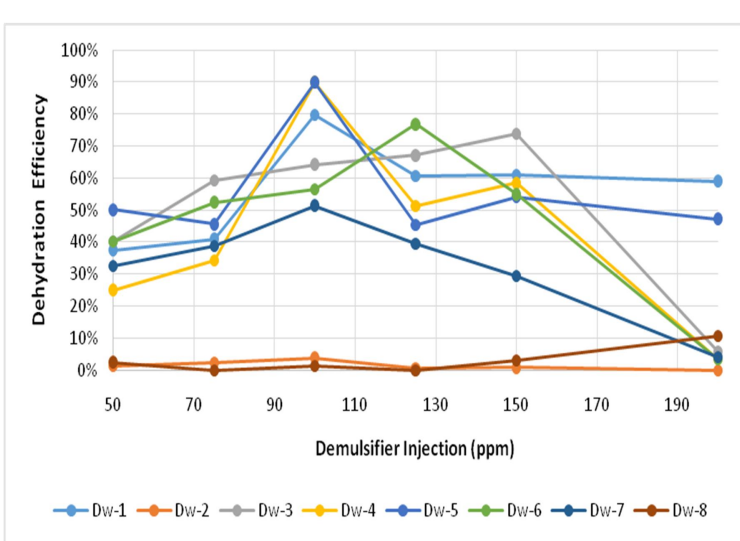

Figure 6. Dehydration efficiency of water base demulsifiers for a residence time of 240 minutes

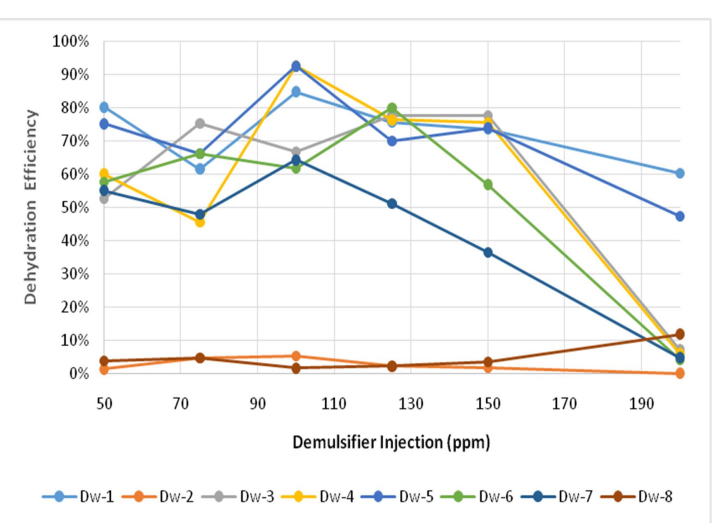

Figure 8. Dehydration efficiency of water base demulsifiers for a residence time of 480 minutes

For long period demulsification, it can be seen that water base demulsifiers performances were approximately same excepting Dw-2, Dw-7, and Dw-8. For short period demulsification, it can be seen that water base demulsifiers performances are different. Dw-2, Dw-4, Dw-7 and Dw-8 were abandoned but Dw-6 at dosage 75 $\mathrm{mg} / \mathrm{L} \sim 150 \mathrm{mg} / \mathrm{L}$ have excellent performance in demulsifiers efficiency curve of water base demulsifiers in $120 \& 240$ minute, especially in 120 minute. Dw-3 also had advantage to Dw-1 \& Dw-5 in demulsifiers efficiency curve of water base demulsifiers in $120 \& 240$. Therefore, Dw-3 and Dw-6 have been chosen as two best water base demulsifiers. Optimal dosage is $125 \mathrm{mg} / \mathrm{L}$.

\section{2-2-Demulsifiers type effect with water cut consideration}

The results of oil/water base demulsifiers study have been shown on figures 9-12, based on different water cuts with 100 ppm dosage in 240 and 480 minute as residence time. 


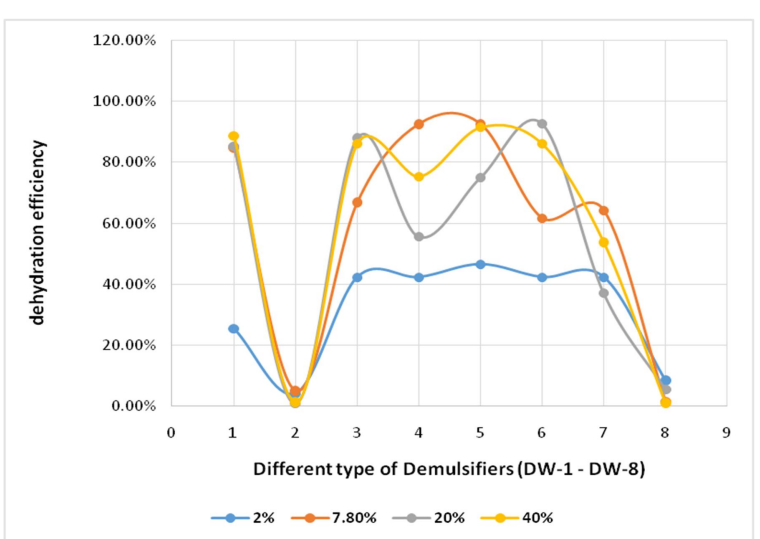

Figure 9. Dehydration efficiency for different water base demulsifiers type in different water cut (100 ppm, 480 minute)

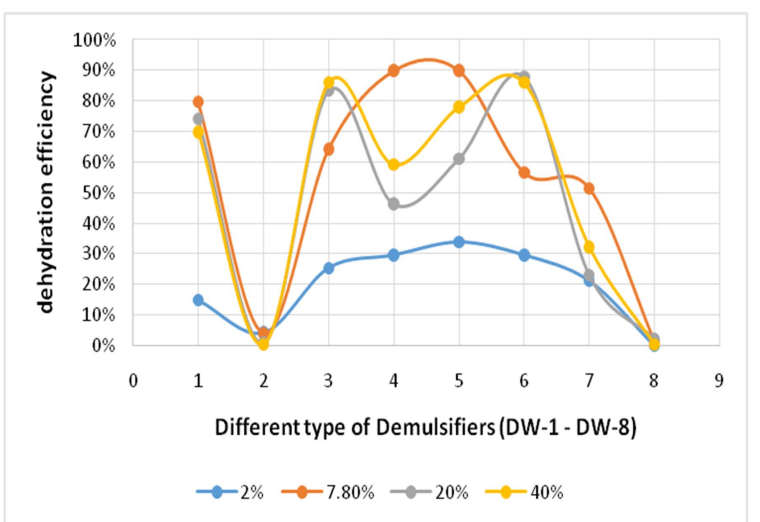

Figure 11. Dehydration efficiency for different water base demulsifiers type in different water cut (100 ppm, 240 minute)

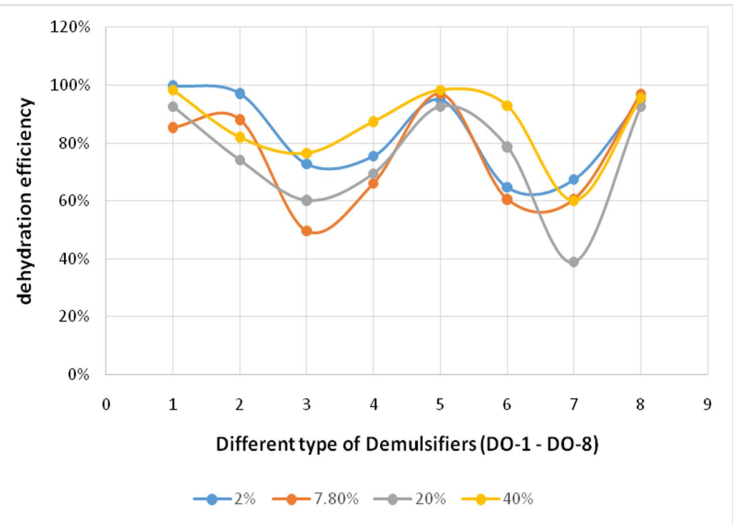

Figure 10. Dehydration efficiency for different oil base de emulsifiers type in different water cut (100 ppm, 480 minute)

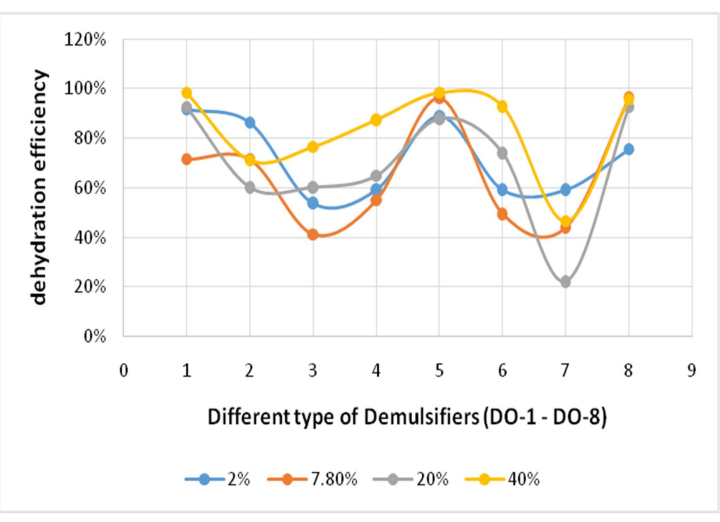

Figure 12. Dehydration efficiency for different oil base de emulsifiers type in different water cut (100 ppm, 240 minute)

The results show that the water base demulsifiers have less efficiency in less crude oil water cuts and it is demonstrated that the water separation by using oil base demulsifiers has a better efficiency. In addition, increasing the residence time has no effect on dehydration efficiency.

\section{3- PARAMETERS INVESTIGATION ON DEHYDRATION EFFICIENCY BY THE SOFTWARE}

The effect of demulsifiers $\mathrm{pH}$ as an independent parameter was studied on demulsifiers performance. For this goal, the Software Qualitec-4 was used.

The Qualitec-4 Software is windows operating software for the Taguchi experimental design technique. It can automatically design experiments based on user specified parameters and levels. It offers variants for experiments, analyzes the results and much more. Table 7 shows the three independent variables' range and levels for the water base demulsifiers investigated in this study. 
Table 7. Experimental range and levels of independent parameters for chosen water base demulsifiers

\begin{tabular}{|l|c|c|c|c|}
\hline \multicolumn{1}{|c|}{ Parameters } & $\begin{array}{c}\text { Dw-1 } \\
(\text { level 1) }\end{array}$ & $\begin{array}{c}\text { Dw-2 } \\
(\text { level 2) }\end{array}$ & $\begin{array}{c}\text { Dw-3 } \\
(\text { level 3) }\end{array}$ & $\begin{array}{c}\text { Dw-6 } \\
(\text { level 4) }\end{array}$ \\
\hline pH & 7.76 & 3.22 & 6.28 & 10.38 \\
\hline Dosage (ppm) & 50 & 75 & 100 & 200 \\
\hline Time (minutes) & 360 & 480 & 600 & 600 \\
\hline
\end{tabular}

The experimental results of each level based on variables for water base demulsifiers (dehydration efficiency) have been mentioned in table 8 .

Table 8 Experimental level dehydration

efficiency for chosen water base demulsifiers

\begin{tabular}{|c|c|}
\hline \multicolumn{2}{|c|}{ Water base demulsifiers } \\
\hline Levels & Efficiency \\
\hline Dw-1 & $70.10 \%$ \\
\hline Dw-2 & $4.56 \%$ \\
\hline Dw-3 & $84.78 \%$ \\
\hline Dw-6 & $4.13 \%$ \\
\hline
\end{tabular}

In this regard, sixteen different runs were performed for water base demulsifiers in order to investigate each parameter effect, as shown in Table 9.

Table 9 Recommended runs by Qualitec-4 software for selected water base demulsifiers

\begin{tabular}{|c|c|c|c|c|}
\hline \multirow{2}{*}{ Run } & \multicolumn{3}{|c|}{ Variables } & \multirow{2}{*}{$\begin{array}{c}\text { Dehydration } \\
\text { efficiency (\%) }\end{array}$} \\
\cline { 2 - 4 } & $\mathrm{pH}$ & Dosage & Time & 70.1 \\
\hline 1 & 7.76 & 50 & 360 & 61.53 \\
\hline 2 & 7.76 & 75 & 480 & 97.62 \\
\hline 3 & 7.76 & 100 & 600 & 60.21 \\
\hline 4 & 7.76 & 200 & 600 & 1.25 \\
\hline 5 & 3.22 & 50 & 480 & 3.42 \\
\hline 6 & 3.22 & 75 & 360 & 17.98 \\
\hline 7 & 3.22 & 100 & 600 & 0 \\
\hline 8 & 3.22 & 200 & 600 & 65.09 \\
\hline 9 & 6.28 & 50 & 600 & 77.48 \\
\hline 10 & 6.28 & 75 & 600 & 66.08 \\
\hline 11 & 6.28 & 100 & 360 & 7.08 \\
\hline 12 & 6.28 & 200 & 480 & 60.09 \\
\hline 13 & 10.38 & 50 & 600 & 70.64 \\
\hline 14 & 10.38 & 75 & 360 & 61.66 \\
\hline 15 & 10.38 & 100 & 480 & 4.13 \\
\hline 16 & 10.38 & 200 & 360 & \\
\hline
\end{tabular}

Based on the results in Table 9, the effective separation for water-based demulsifiers occurred in experiment 3 with about $97 \%$ dehydration efficiency, while in experiment 8 , the water separation was $0 \%$, being at the end of the list. In these runs, the mean separation rate was about $45.3 \%$. 
Table 10 shows that $\mathrm{pH}$ is the most influential parameter in separating water $(56.079 \%)$, while time had the lowest effect on separation, around $9.8 \%$. The total error for the results of the above experiments is $10.1 \%$.

Table 10. Investigation of variances for chosen water base demulsifiers

\begin{tabular}{|l|c|c|c|c|c|c|}
\hline Factors & DOF & $\begin{array}{c}\text { Sum of } \\
\text { sqrs. }\end{array}$ & Variance & $\begin{array}{c}\mathrm{F} \\
\text { ratio }\end{array}$ & Pure sum & $\begin{array}{c}\text { Percent } \\
(\%)\end{array}$ \\
\hline $\mathrm{pH}$ & 3 & 9571.326 & 3190.442 & 28.767 & 9238.613 & 56.079 \\
\hline Dosage & 3 & 4290.921 & 1430.307 & 12.896 & 3958.209 & 24.026 \\
\hline Time & 3 & 1946.519 & 648.839 & 5.85 & 1613.807 & 9.795 \\
\hline Total & 15 & 665.423 & & & & 10 \\
\hline
\end{tabular}

The information about the selected parameter is shown in Table 11. In this table, the optimal value related to each factor, corresponding to its level, was revealed.

Table 11. The optimum levels for all selected water base demulsifiers

\begin{tabular}{|c|c|c|c|c|}
\hline No. & Factors & Level description & Level & Contribution \\
\hline 1 & $\mathrm{pH}$ & 7.76 & 1 & 27.092 \\
\hline 2 & Dosage & 100 & 3 & 15.562 \\
\hline 3 & Time & 600 & 3 & 13.065 \\
\hline
\end{tabular}

\section{CONCLUSIONS}

A screening process was used to choose the most efficient demulsifiers, taking into account their price. The oil base demulsifiers have better dehydration efficiency, about $96 \%$ based on water/oil base results. Using this type of demulsifiers, cover crude oil with wide water cut range (2\%-40\%). So the demulsifiers Do-5, Do- 8 and Do- 1 , have chosen as three of the best oil-based demulsifiers. The optimal dosages are $100 \mathrm{ppm}$, $100 \mathrm{ppm}$ and $200 \mathrm{ppm}$, respectively.

In the second part of the research, because water-based demulsifiers have a lower water separation efficiency, Qualitec-4 software investigates the effect of the $\mathrm{pH}$ parameter and the best efficiency for water-based demulsifiers. This is obvious: the water separation percentage using water base/oil base demulsifiers varies, and the difference in separation depends on dosage, residence time, and demulsifiers properties such as $\mathrm{pH}$.

The result shows that the $\mathrm{pH}$ of demulsifiers can affect about $27 \%$ on dehydration efficiency, and other conditions such as dosage and residence time have less impact. In this regard, selecting the water base demulsifier with $\mathrm{pH}=7.76$, dosage $=100$, time $=600$, has the best performance (approximately 100\% dehydration efficiency considering 10\% error).

\section{REFERENCES}

[1] Manning, F. S., Thompson, R.E., Oil field Processing Vol. 2: Crude Oil, Penn Well Books, 1995. 
[2] Sjoblom, J., Hemmingsen, P. V., Kallevik, H., The Role of Asphaltenes in Stabilizing Water in Crude Oil Emulsions, In: O. C., Mullins, E. Y., Sheu, A., Hammami, A. G., Marshall, (Eds.), Asphaltenes, Heavy Oils, and Petroleomics, Springer, New York, p. 549, 2007.

[3] Jones, T. J., Neustadter, E. L., Wittingham, K. P., Water-in-crude oil emulsion stability and emulsion destabilization by chemical demulsifiers. J. Can. Pet. Tech. vol. 17, April, 1978.

[4] Poteau, S., Argillier, J. F., Langevin, D., Pincet, F., Perez, E., Influence of pH on stability and dynamic properties of asphaltenes and other amphiphilic molecules at the oil-water interface, Energy \& Fuels, no. 19, pp.1337-1341, 2005.

[5] Strassner, J. E., Effect of $\mathrm{pH}$ on interfacial films and stability of crude oil-water emulsions, JPT 303, March, 1968.

[6] Daaou, M., Bendedouch, D., Bouhadda, Y., Vernex-Loset, L., Modaressi, A., Rogalski, M., Explaining the flocculation of Hassi Messaoud asphaltenes in terms of structural characteristics of monomers and aggregates, Energy \& Fuels, no. 23, pp. 5556- 5563, 2009.

[7] Fortuny, M., Oliveira, C. B. Z., Melo, R. L. F. V., Nele, M., Coutinho, R. C. C., Santos, A. F., Effect of salinity, temperature, water content, and $\mathrm{pH}$ on the microwave demulsification of crude oil emulsions, Energy Fuels, no. 21, pp. 1358-1364, 2007.

[8] Moradi, M., Alvarado, V., Huzurbazar, S., Effect of salinity on water-in-crude oil emulsion: Evaluation through drop-size distribution proxy, Energy \& Fuels, no. 25, pp.260-268, 2011. 